\title{
Plowing-Induced Structuring of Compliant Surfaces
}

\author{
Juan J. Mazo, ${ }^{1,2}$ Pedro J. Martínez, ${ }^{2,3}$ Patricia Pedraz, ${ }^{4}$ Jana Hennig, ${ }^{5}$ and Enrico Gnecco ${ }^{5}$ \\ ${ }^{1}$ Departamento de Física de la Materia Condensada, Universidad de Zaragoza, Zaragoza, Spain \\ ${ }^{2}$ Instituto de Ciencia de Materiales de Aragón, CSIC-Universidad de Zaragoza, Zaragoza, Spain \\ ${ }^{3}$ Departamento de Física Aplicada, Universidad de Zaragoza, Zaragoza, Spain \\ ${ }^{4}$ KTH Royal Institute of Technology, Stockholm, Sweden \\ ${ }^{5}$ Otto Schott Institute of Materials Research (OSIM), Friedrich Schiller University Jena, \\ Löbdergraben 32, 07743 Jena, Germany
}

(Received 13 November 2018; revised manuscript received 12 March 2019; published 25 June 2019)

\begin{abstract}
The structures produced by a sharp tip scraping a compliant surface are modeled in the illustrative case of scan patterns formed by a series of parallel lines. This is made possible by a modified version of the Prandtl model for stick-slip friction, with an interaction energy landscape replicating the morphology of the evolving surface. As a result, a ripple motif emerges with a tilt angle increasing linearly with the distance between the scan lines, except for the region close to the left boundary of the scanned area, where the ripples are oriented at $90^{\circ}$. This region can penetrate considerably to the right, forming a complex branched pattern. These predictions are substantiated by atomic force microscopy nanolithography experiments on polystyrene surfaces at room temperature. A simple and robust theoretical protocol for reproducing earlystage wear processes (potentially going beyond single contacts) is thus made available.
\end{abstract}

DOI: 10.1103/PhysRevLett.122.256101

Generally speaking, when the shear deformation in a junction formed between two rough surfaces sliding past each other reaches a threshold value, the junction gets broken and a new one is formed along the direction of sliding [1]. When the contact area has atomic-scale dimensions, this statement can be made more precise within the theoretical model developed by Ludwig Prandtl in the end of 1920s [2]. In the Prandtl model, the stick-slip motion of a sharp tip sliding on a crystal surface is associated to a sawtooth variation of the lateral (friction) force acting on the tip, which literally jumps from an equilibrium position to another one on the lattice. Several analytic and numerical relations between static and kinetic friction forces, lattice symmetries $[3,4]$, sliding velocity and temperature [5,6], and stiffness and surface potentials [7], including time variations of the last ones [8-10], have been derived on this basis, and confirmed experimentally. Extensions to larger contact areas are in the focus of recent literature $[11,12]$ and it may be only a question of time until applications to rough surfaces of technical interest will be found. Nevertheless, the Prandtl model is based on the hypothesis that the mechanical response of the tip-surface junction is elastic up to its rupture. Plastic deformation and abrasive wear, which often accompany sliding friction, are not considered in the model and in most of the aforementioned extensions, with the noticeable exception of the work of Filippov et al. on the wear of ionic crystals associated to atom-by-atom displacement caused by the sliding tip [13].

In this Letter we discuss how the Prandtl model can be successfully extended to predict the occurrence of periodic surface patterns accompanying the stick-slip motion of a tip on a compliant surface. Our approach, which generalizes previous work on two-dimensional (2D) profiles [14], starts from the assumption that, under the pressure exerted in the stick phase, the surface conforms plastically to the tip shape, and acts at the same time as an effective energy landscape for the lateral motion of the tip elastically driven along the surface. Depending on the scan conditions, a variety of structures can be produced, but to test the validity of the model we have limited our analysis to the exemplary case of a series of parallel lines. This choice is suited for comparison with friction force maps of crystal surfaces acquired by atomic force microscopy (AFM) and well reproduced by the standard Prandtl model [15]. In the present case, the experimental images are obtained from nanolithography experiments on polystyrene, a model material for AFM-induced surface modifications [16].

The samples were prepared from polystyrene pellets with high molecular weight (Polymer Source and SigmaAldrich with MW > 320000) dissolved in toluene (Merk 99.9\% HPLC) with a concentration of $6 \mathrm{wt} \%$. Polymer films were obtained by spin coating on silicon substrates previously cleaned by sonication in acetone, in ethanol, and in ultrapure water (and dried with air flow after each step). The process was carried out using a WS-400B-6NPP-LITE spin coater (Laurell Technologies Corporation, USA) at $3000 \mathrm{rpm}$ for $60 \mathrm{~s}$. The chosen values correspond to an expected film thickness of about $400 \mathrm{~nm}$ [17]. Finally, the samples were allowed to dry at room temperature for $20 \mathrm{~h}$ before performing the nanolithography measurements with 

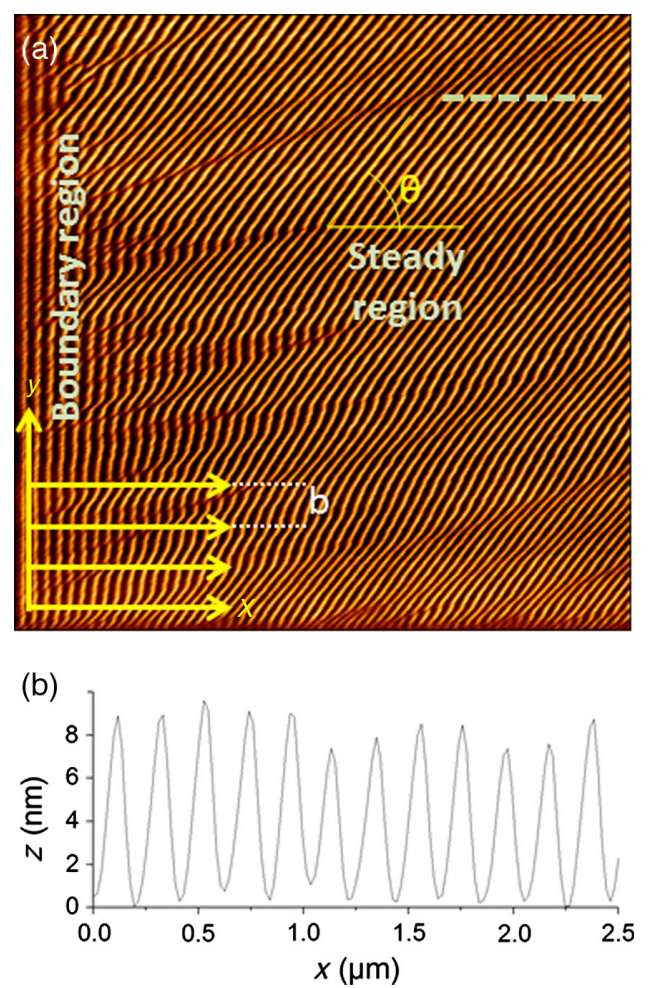

FIG. 1. (a) AFM topography image of a polystyrene surface being scraped by a sharp silicon tip sliding along the path shown in the bottom left corner. The frame size is $10 \times 10 \mu \mathrm{m}^{2}$, and the normal force, scan velocity, and separation distance have, respectively, the following values: $b=20 \mathrm{~nm}, F_{N}=200 \mathrm{nN}$, and $v=11.2 \mu \mathrm{m} / \mathrm{s}$. (b) Cross section corresponding to the dashed line in Fig. 1(a).

a NanoWizard 4 AFM (JPK Instruments, Berlin) operated in contact mode with FMG01 silicon probes (NT-MDT, Moscow). As a scan pattern, we chose a series of parallel scan lines, separated by a distance $b$.

A typical result is shown in Fig. 1(a). Close to the left edge of the scanned area a ripple pattern aligned at $90^{\circ}$ with respect to the fast $(x)$ direction can be easily distinguished. This region breaks up into several branches and disappears into the second region, where a pattern of ripples regularly oriented with an angle $\theta<90^{\circ}$ is observed. The same pattern is visible in the corresponding lateral force image (Fig. S1 in the Supplemental Material [18]). The ripple amplitude, in the order of $10 \mathrm{~nm}$, is well above the maximum corrugation of the pristine surface $(0.7 \mathrm{~nm}$ only, as seen in Fig. S2 in the Supplemental Material [18]).

The values of $\theta$ as a function of $b$ as obtained in different series of measurements are plotted in Fig. 2. The angle $\theta$ increases with $b$, and the two regions become indistinguishable when $b \approx 40 \mathrm{~nm}$. The lateral force oscillates by approximately $200 \mathrm{nN}$ when the ripples are formed, suggesting a stick-slip motion. The application of the Prandtl model for reproducing the structuring process appears well-founded.

In 3D the interpretation begins by considering a perfectly flat surface $z(\mathbf{r})=0$, where $\mathbf{r}$ is a generic location on the

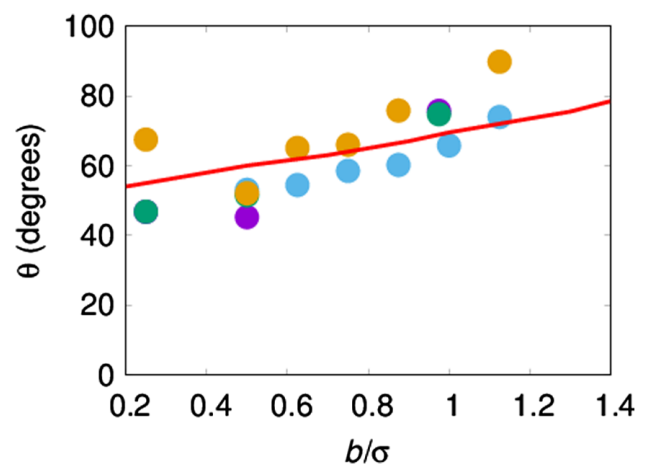

FIG. 2. Ripple orientation far from the left boundary as a function of the separation distance $b$. The circles (referred to $\sigma=40 \mathrm{~nm}$ ) correspond to distinct series of AFM measurements (considering the waviness of the ripples caused by the branching effect, an error in the order of few percent should be taken into account). The red line $(\sigma=50 \mathrm{~nm})$ corresponds to the results of the numeric simulations, see Fig. 3.

$x y$ plane and the $z$ axis is oriented outwards perpendicular to that plane. When the tip apex is located at a given position, $\mathbf{r}_{0}$, the surface profile is modified according to the equation

$$
\frac{\partial z(\mathbf{r}, t)}{\partial t}=N\left(z_{0}\right) \Psi\left(\left|\mathbf{r}-\mathbf{r}_{0}\right|\right)
$$

where the indentation rate $N\left(z_{0}\right)$, with $z_{0}$ the height of the tip apex, decays exponentially with a length constant $\lambda$ as the tip penetrates the substrate (see Supplemental Material [18]). The function $\Psi$ corresponds to a Gaussian shaped pit surrounded by a circular rim also having a Gaussian profile:

$$
\Psi(\rho)=-e^{-\rho^{2} / 2 \sigma^{2}}+\alpha e^{-(\rho-2 \sigma)^{2} / 2 \sigma^{2}} .
$$

These assumptions are consistent with the shape and time evolution of the pits typically observed on polymer surfaces indented by AFM [14]. The coefficient $\alpha \approx 0.2$ is chosen in such a way that the total volume under the surface remains constant, which is the case if the deformation is plastic. The next key assumption is that the evolving surface profile acts as an effective potential $U_{\mathrm{int}}(\mathbf{r}, t)$ for the tip-surface interaction with $U_{\text {int }}(\mathbf{r}, t) \propto z(\mathbf{r}, t)$.

While the tip is indenting the surface, it is also pulled laterally by its support, which, in the case of AFM, corresponds to the clamped end of an elastic microcantilever moving along the $x$ axis with a constant velocity $v$. Note that the plane coordinates of tip and support are not necessarily the same, and that one has to distinguish between two values of the spring constants, one along the scan direction, $k_{x}$, and another perpendicular to it, $k_{y}$. These quantities define the effective stiffness of contact and cantilever and, a priori, they are not equal. The evolving potential $U_{\text {tot }}$ given by the sum of $U_{\text {int }}$ and the elastic energy $U_{\mathrm{el}}$ associated to the spring deformation is finally used to define the total force acting on the tip, the equation of motion of which thus reads 


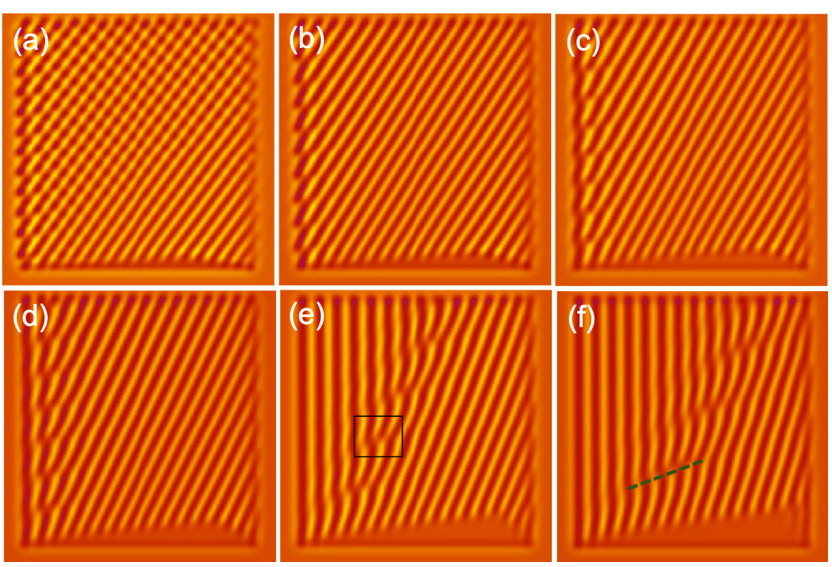

FIG. 3. (a)-(f) Surface patterns obtained from the Prandtl model for stick-slip friction described in the text. The ratio $b / \sigma$ has been varied from 0.2 to 1.2 by steps of 0.2 . Each frame has a linear size of $4 \mu \mathrm{m}$. The rectangle in (e) corresponds to the region showed in Fig. 4(a). The dashed line in (f) shows the branching also seen experimentally, Fig. 1(a). Note that the color scale has been adjusted to have the same contrast in all images.

$$
\Gamma \frac{d \mathbf{r}_{0}}{d t}=-\left.\nabla U_{\text {tot }}(\mathbf{r}, t)\right|_{\mathbf{r}=\mathbf{r}_{0}} .
$$

The equations for the tip dynamics and the surface height so defined have been solved numerically in a range of parameter values typical for AFM experiments, i.e., $\sigma=50 \mathrm{~nm}, \quad k_{x}=2 \mathrm{~N} / \mathrm{m}, \quad k_{y}=10 \mathrm{~N} / \mathrm{m}, v=10 \mu \mathrm{m} / \mathrm{s}$, $N=100 \mathrm{~nm} / \mathrm{s}$ (corresponding to an energy variation rate of $6 \times 10^{5} \mathrm{nNnm} / \mathrm{s}$ consistent with the measured lateral force values) and $\lambda=1.8 \mathrm{~nm}$ (see Supplemental Material [18] for additional details). The results obtained for different values of $b / \sigma$ between 0.2 and 1.2, are shown in Fig. 3. In the scan process, the tip sticks to the surface and forms a "crater" according to Eq. (2), which, at the same time, is pulled to the right and, to a minor extent, to the top of the scanned area, by a spring force increasing with time. As in the standard Prandtl mechanism, the tip moves slightly to the right while it is pulled till an elastic instability is reached. At this point it suddenly slips into the next equilibrium position and the stick-slip process repeats. This can be appreciated by plotting the components of the lateral force along $x$ and $y$ direction, together with the tip path, as done in Fig. 4. When a new scan line is started, the tip motion is guided by the surface pattern formed up to that point. Due to the slight shift of the tip position in the stick phase, the resulting ripple structures are inclined by a certain angle $\theta$. In the present case, the larger the $b$ the lower the overlap of the indentation print with the surface pattern already existing. As a consequence, $\theta$ increases with $b$. When $b \gtrsim \sigma$, a steady value of $\theta$ is reached only after a sequence of vertical ripples propagating more and more deeply to the right as the number of scans increases. The transition occurs through a region where the ripples oscillate between $\theta$ and $90^{\circ}$ giving rise to a series of parallel
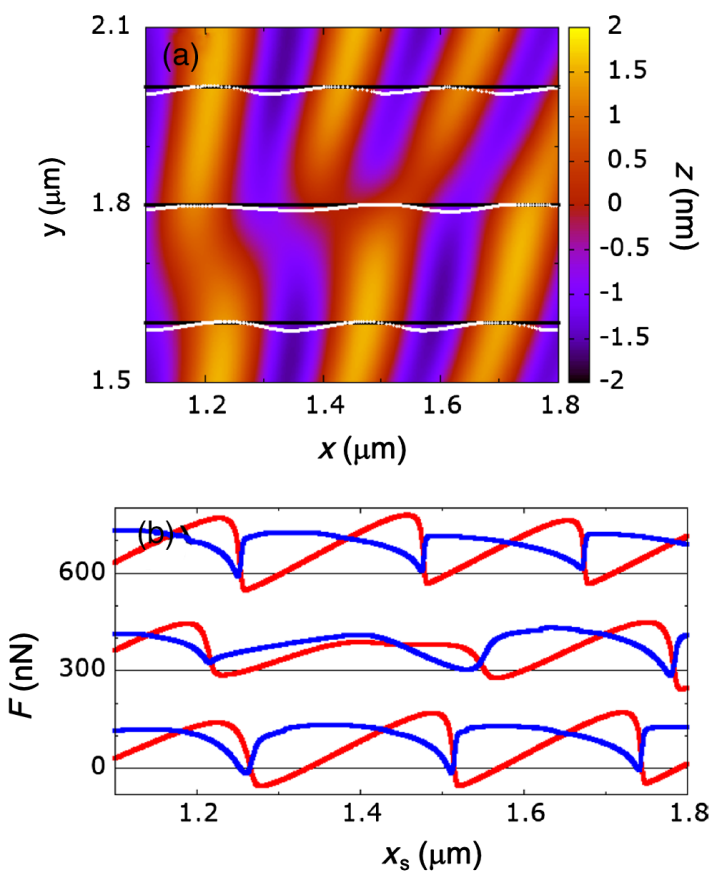

FIG. 4. (a) Detail pattern in Fig. 3(e) showing the support (black) and the tip (white) paths corresponding to three nonconsecutive scan lines. (b) $x$ (red) and $y$ (blue) components of the lateral force computed while scanning the black lines in (a) left to right. Note that the stick phase is interrupted at the bifurcation point, where the equilibrium becomes neutral and the tip follows the support for approximately $300 \mathrm{~nm}$.

straight lines with lower inclination than $\theta$, like that shown (in green) in Fig. 3(f). Furthermore, the ripple formation is difficult in the very first scan lines, and the ripples may not appear at all in a limited region in the bottom part of the scanned areas. These features can be also recognized in the AFM image in Fig. 1(a). The increase of the ripple angle with $b$ observed in the simulations is compared with our experimental results in Fig. 2, red line versus solid circles, proving that the proposed model can adequately reproduce the formation of plowing-induced structures on a compliant surface.

Note that, in the simulations, the ripples are only obtained if $k_{y} \gg k_{x}$, consistently with the fact that the lateral stiffness of the cantilever associated to its buckling (in the $y$ direction) is much larger than the lateral stiffness associated to its torsion (along $x$ ) [19]. In order to support this assumption, we have also tried to pattern the polymer surface with the fast scan direction oriented along the $y$ axis and, as a result, only an irregular array of pits was observed but no ripples, as seen in Fig. S3 in the Supplemental Material [18]. However, the previous inequality does not hold for atomic-scale friction measurements with loading forces in the $\mathrm{nN}$ range. In that case, the deformation is dominated by the few atoms in the sliding contact and, as suggested by a comparison of the experimental data with the standard Prandtl model [20], it was rather concluded that $k_{y} \approx k_{x}$. 

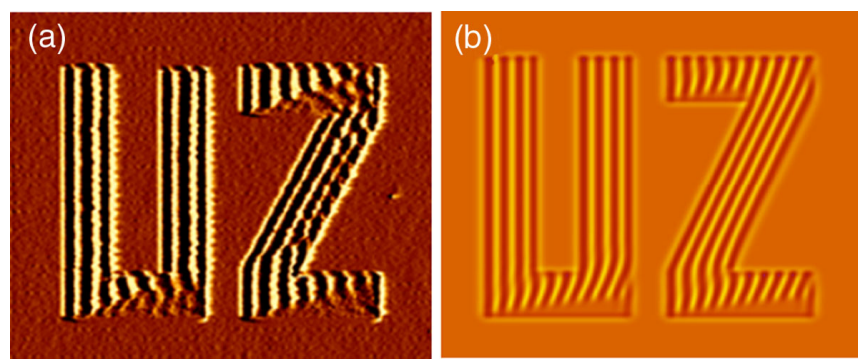

FIG. 5. (a) Experimental and (b) numerical ripple patterns obtained when the loading force is applied only in two areas corresponding to the letters $U$ and $Z$. Frame sizes: $4.8 \times 3.9 \mu \mathrm{m}^{2}$.

Finally, we have also studied the ripples obtained with different shapes of the scanned areas or with the horizontal lines scanned forward and backward. In the first case, we have scraped two patches resembling the letters $U$ and $Z$. Since the width of these patches correspond to three periods only, it is not surprising that, in both experiments and simulations, the ripples are oriented parallel to the left edges in most of the scanned area (Fig. 5). This result shows that the ripple orientation is controlled by the geometry of the scanned area only, if this is narrow enough, which is important in view of possible applications to nanooptical gratings produced by scanning nanolithography.

On the other hand, the patterns obtained when scanning in both directions are quite puzzling. As seen in Fig. 6 the ripple orientation is flipped horizontally in this case, in agreement with previous reports in the literature [21]. This effect is not seen in the simulations, leading to vertical ripples in case of bidirectional scanning, Fig. 7(a), which has been also reported experimentally, when the lines are alternately scanned forward and backward [22]. A possible explanation is that the stiffness beneath the tip apex is changed when the polymer is scraped, which would

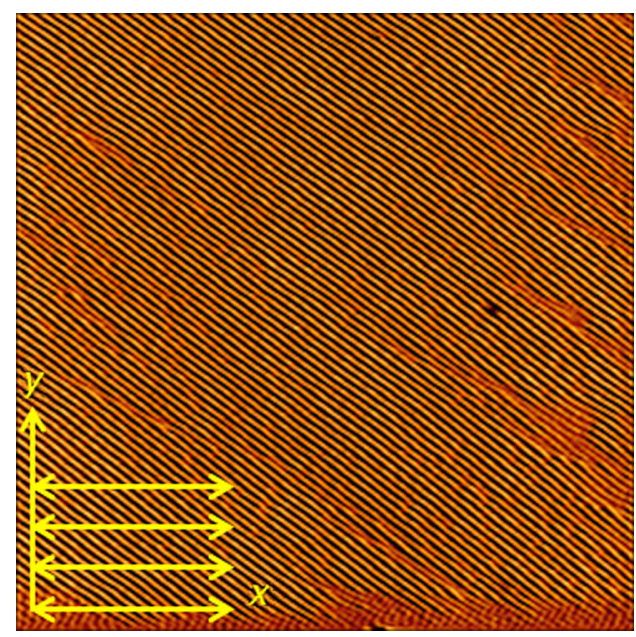

FIG. 6. Same as Fig. 1(a) with a bidirectional scan path and the parameter values $b=10 \mathrm{~nm}, F_{N}=100 \mathrm{nN}$ and $v=22.6 \mu \mathrm{m} / \mathrm{s}$ (this figure was imaged while scanning left to right). introduce a substantial asymmetry when the tip follows precisely the same path on the way back. At the molecular level, a lower stiffness may result from a combination of brushing, combing and chain scission phenomena, which have been observed in molecular dynamic (MD) simulations of polymer friction [23]. Since MD simulations of polymer surfaces sheared by a silicon tip are, at a singlechain level, not available, we have performed additional simulations, assuming that $k_{x}$ is simply reduced by a factor of 2 on the way back. This means that, at the turning point, the tip spends more time in the last pit of the ripple pattern than in the forward scan, and the final shape of this pit is not symmetric. The distorsion is propagated over the whole path backward, and results in an orientation of the ripples well above $90^{\circ}$, as seen in Fig. 7(b).

To summarize, we have introduced a simulation protocol, which is physically based on the Prandtl mechanism and is able to reproduce the surface modifications occurring in a compliant material scraped by a sharp tip, as attested by a comparison of the simulated patterns with AFM measurements on polystyrene. It is worth to mention that we have also checked the robustness of our results performing simulations from initially rough surfaces and adding thermal fluctuations in the system equations (Fig. S5 in the Supplemental Material [18]). The model has some limitations which could be reasonably overcome. Different indentation prints (e.g., pyramidal ones) could be introduced to test the importance of the tip face orientation suggested by recent experiments by Yan et al. [24]. Depending on the experimental conditions, the indentation profile could also change (i.e., getting broader and more shallow) with the penetration depth, till the irreversibly deformed area stops growing. With these issues taken into account, an important follow-up of this Letter may consist in considering an ensemble of randomly distributed tips, and simulating the evolution of surface structures typically observed in multiple-contact abrasive wear processes.
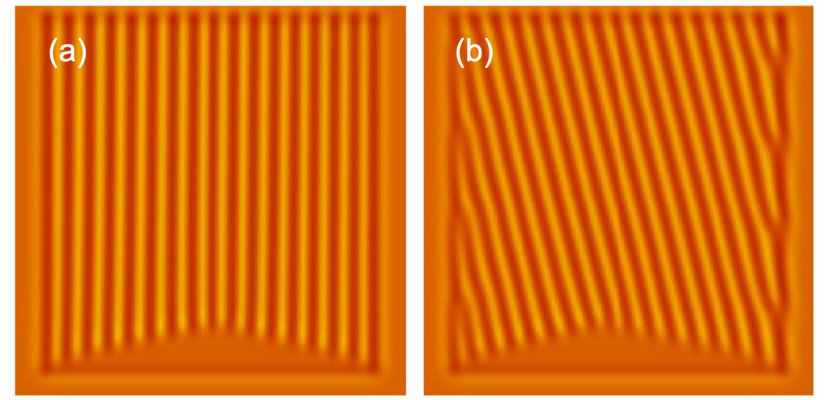

FIG. 7. Simulated ripples with bidirection scan path. The lateral contact stiffness is (a) kept at the constant value $k_{x}=2 \mathrm{~N} / \mathrm{m}$, (b) alternated between $k_{x}=3 \mathrm{~N} / \mathrm{m}$ in the forward direction and $k_{x}=1.5 \mathrm{~N} / \mathrm{m}$ backwards. The other parameter values are: $b=20 \mathrm{~nm}, v=20 \mu \mathrm{m} / \mathrm{s}, N=50 \mathrm{~nm} / \mathrm{s}, k_{y}=10 \mathrm{~N} / \mathrm{m}$. Frame size: $4 \times 4 \mu \mathrm{m}^{2}$. 
J. J. M. and P. J. M. acknowledge support from Spanish Ministerio de Economía, Industria y Competitividad (MINECO) Projects No. FIS2014-55867-P and No. FIS2017-87519-P cofinanced by the Fondo Europeo de Desarrollo Regional (FEDER), and by the Gobierno de Aragón, Grant No. E36_17R to the FENOL group.

[1] F. P. Bowden and D. Tabor, Friction and Lubrication of Solids (Oxford University Press, New York, 1950).

[2] L. Prandtl, Z. Angew. Math. Mech. 8, 85 (1928).

[3] P. Steiner, R. Roth, E. Gnecco, A. Baratoff, and E. Meyer, Phys. Rev. B 82, 205417 (2010).

[4] E. Gnecco, Europhys. Lett. 91, 66008 (2010).

[5] Y. Sang, M. Dubé, and M. Grant, Phys. Rev. Lett. 87, 174301 (2001).

[6] M. H. Müser, Phys. Rev. B 84, 125419 (2011).

[7] O. Y. Fajardo and J. J. Mazo, Phys. Rev. B 82, 035435 (2010).

[8] R. Roth, O. Y. Fajardo, J. J. Mazo, E. Meyer, and E. Gnecco, Appl. Phys. Lett. 104, 083103 (2014).

[9] J. J. Mazo, D. Dietzel, A. Schirmeisen, J. G. Vilhena, and E. Gnecco, Phys. Rev. Lett. 118, 246101 (2017).

[10] K. Tian, D. L. Goldsby, and R. W. Carpick, Phys. Rev. Lett. 120, 186101 (2018).

[11] M. M. van Wijk, M. Dienwiebel, J. W. M. Frenken, and A. Fasolino, Phys. Rev. B 88, 235423 (2013).

[12] S. G. Balakrishna, A. S. de Wijn, and R. Bennewitz, Phys. Rev. B 89, 245440 (2014).
[13] A. E. Filippov, V. L. Popov, and M. Urbakh, Phys. Rev. Lett. 106, 025502 (2011).

[14] E. Gnecco, P. Pedraz, P. Nita, F. Dinelli, S. Napolitano, and P. Pingue, New J. Phys. 17, 032001 (2015).

[15] G. Fessler, I. Zimmermann, Th. Glatzel, E. Gnecco, P. Steiner, R. Roth, T. D. Keene, S. X. Liu, S. Decurtins, and E. Meyer, Appl. Phys. Lett. 98, 083119 (2011).

[16] O. N. Leung and M. C. Goh, Science 255, 64 (1992).

[17] D. B. Hall, P. Underhill, and J. M. Torkelson, Polym. Eng. Sci. 38, 2039 (1998).

[18] See Supplemental Material at http://link.aps.org/ supplemental/10.1103/PhysRevLett.122.256101 for friction force variations accompanying the rippling process, topography map of pristine surface, comparison of scratching parallel and perpendicular to the cantilever axis, role of initial roughness, and details about the performed numerical simulations.

[19] Springer Handbook of Nanotechnology, edited by B. Bhushan (Springer, Berlin, 2010).

[20] P. Steiner, R. Roth, E. Gnecco, A. Baratoff, S. Maier, T. Glatzel, and E. Meyer, Phys. Rev. B 79, 045414 (2009).

[21] S. Napolitano, M. D'Acunto, P. Baschieri, E. Gnecco, and P. Pingue, Nanotechnology 23, 475301 (2012).

[22] Y. Yan, Y. Sun, J. Li, Z. Hu, and X. Zhao, Nanoscale Res. Lett. 9, 372 (2014).

[23] L. Dai, M. Minn, N. Satyanarayana, S. K. Sinha, and V. B. C. Tan, Langmuir 27, 14861 (2011).

[24] Y. Yan, X. Cui, Y. Geng, and Y. He, Micro Nano Lett. 12, 1011 (2017). 